\title{
TAXONOMÍA Y DISTRIBUCIÓN DE MELOE (EURYMELOE) SAHARENSIS CHOBAUT, 1898 (COLEOPTERA, MELOIDAE), CON NUEVAS SINONIMIAS Y PRIMEROS REGISTROS PARA EUROPA Y LA MACARONESIA
}

\author{
J. L. Ruiz ${ }^{1}$, M. A. Bologna ${ }^{2}$ \& M. García-París 3* $^{3^{*}}$
}

\begin{abstract}
RESUMEN
J. L. Ruiz, M. A. Bologna \& M. García-París. 2010. Taxonomía y distribución de Meloe (Eurymeloe) saharensis Chobaut, 1898 (Coleoptera, Meloidae), con nuevas sinonimias y primeros registros para Europa y la Macaronesia. Grael/sia, 66(1): 85-96.

Entre las especies más raras de la familia Meloidae en la cuenca mediterránea destaca un pequeño grupo de especies del género Meloe relacionadas con M. saharensis Chobaut, 1898. El estudio taxonómico de nueve ejemplares (incluido el material tipo de M. saharensis, M. otini Peyerimhoff, 1949 y M. vignai Bologna, 1990), permite concluir que las diferencias específicas señaladas previamente entre $M$. saharensis, M. otini y M. marianii Kaszab, 1983, en especial la visibilidad del metanoto, entran dentro de la variabilidad de una única especie. El estudio de un conjunto más amplio de caracteres (incluida la genitalia masculina) tampoco permite la separación de taxones, excepto en el caso de $M$. vignai. Por lo tanto se propone formalmente la sinonimia de M. marianii (syn. nov.) y M. otini (syn. nov.) con M. saharensis, de forma que este complejo de especies queda reducido a únicamente dos taxones: $M$. saharensis y $M$. vignai. Los ejemplares examinados permiten atribuir una amplia distribución geográfica a M. saharensis, que se extendería desde Israel y Arabia Saudí hasta las Islas Canarias (Tenerife), con presencia en el sureste de España y en la mayor parte de los países norteafricanos (Egipto, Túnez, Argelia y Marruecos). A pesar de su antigüedad, ya que data de hace al menos 80 años, el registro de $M$. saharensis en Murcia constituye el primer dato conocido sobre la presencia de esta especie en Europa.
\end{abstract}

Palabras clave: Taxonomía; Morfología; Meloidae; Meloe saharensis; Meloe vignai; Nuevas Sinonimias; Meloe otini; Meloe marianii; Región Mediterránea; Zonas áridas; Norte de África; España.

\section{ABSTRACT}

J. L. Ruiz, M. A. Bologna \& M. García-París. 2010. Taxonomy and geographic distribution of Meloe (Eurymeloe) saharensis Chobaut, 1898 (Coleoptera, Meloidae), with new synonymies and first records for Europe and Macaronesia. Graellsia, 66(1): 85-96 (in Spanish).

A small group of species of the genus Meloe related to $M$. saharensis Chobaut, 1898 stands out amongst the rarest species of the family Meloidae in the Mediterranean Basin. The

Instituto de Estudios Ceutíes. Paseo del Revellín, 30. Apartado 593. 51080 Ceuta. España.

Dipartimento di Biologia Ambientale. Università degli Studi "Roma Tre", Viale G. Marconi 446. 00146 Roma. Italia.

Museo Nacional de Ciencias Naturales. CSIC. José Gutiérrez Abascal, 2. 28006. Madrid. España.

* Autor para correspondencia: mparis@mncn.csic.es 


\begin{abstract}
taxonomic study of nine specimens (including type material of $M$. saharensis, $M$. otini Peyerimhoff, 1949 and M. vignai Bologna, 1990), allows us to conclude that previously reported specific differences between $M$. saharensis, M. otini and M. marianii Kaszab, 1983, particularly the appearance of the metanotum, fall within the variability of a single species. The study of a broader set of characters, including male genitalia, does not contribute to the separation of taxa except for $M$. vignai. Consequently, we propose the formal synonymy of $M$. marianii (syn. nov.) and M. otini (syn. nov.) with $M$. saharensis, and therefore this species complex is reduced to two taxa: $M$. saharensis and M. vignai. Specimens examined allow us to assign a broad geographic range to $M$. saharensis, which extends from Israel and Saudi Arabia to the Canary Islands (Tenerife), with presence in southeastern Spain and most of the northern African countries (Egypt, Tunisia, Algeria and Morocco). Despite its antiquity, dating back more than 80 years, the record of $M$. saharensis in Murcia represents the first known data about its presence in Europe.
\end{abstract}

Key-words: Taxonomy; Morphology; Meloidae; Meloe saharensis; Meloe vignai; New Sinonymy; Meloe otini; Meloe marianii; Mediterranean Region; Arid zones; Northern Africa; Spain.

\section{Introducción}

Uno de los grupos de taxonomía más complicada dentro del amplio género Meloe Linnaeus, 1758, está constituido por el subgénero Eurymeloe Reitter, 1911, que incluye unas 50 especies de distribución predominantemente paleártica (Pinto \& Selander, 1970; Bologna, 1988, 1990, 1991, 2008a; Bologna et al., 1989). En el ámbito del subgénero Eurymeloe, y dentro del grupo de Meloe rugosus Masham, 1802, Bologna (1990) estableció un complejo de especies, al que nos referiremos de aquí en adelante como el complejo de $M$. saharensis, integrado por Meloe (Eurymeloe) marianii Kaszab, 1983, Meloe (Eurymeloe) otini Peyerimhoff, 1949, Meloe (Eurymeloe) saharensis Chobaut, 1898 y Meloe (Eurymeloe) vignai Bologna, 1990. Este conjunto de taxones.

La única información taxonómica y geográfica conocida del complejo de $M$. saharensis se limitaba casi exclusivamente a la utilizada en las descripciones originales. Así, Meloe saharensis fue descrita a partir de una hembra, procedente de Ghardaia, en el Sáhara centro-septentrional argelino (Chobaut, 1898). Meloe otini se describió a partir de un macho procedente de la meseta de Guercif ("plaine de Guercif"), en Marruecos nororiental (Peyerimhoff, 1949); Kocher $(1954,1956)$ añade a esta especie las localidades de Oujda, $140 \mathrm{~km}$ al este de Guercif (véase Bologna, 1988: 241) y la meseta situada al este de Outat-el-Hajj, aproximadamente $100 \mathrm{~km}$ al suroeste de la localidad-tipo. Meloe marianii fue descrita por Kaszab (1983) a partir del estudio de dos machos: el holotipo procedente de Alejandría, Egipto y un paratipo de Khashm Khasf, en Arabia Saudí; Bologna (1988) amplía la descripción morfológica de Kaszab (1983) y añade dos nuevos registros del norte de Egipto basados en otros tantos ejemplares (Marsa Matrouth e Ikingi Mariout); recientemente, Bologna (2008a) señala la presencia de M. marianii en Israel, aunque sin precisar localidad. La descripción original de Meloe vignai está basada en el holotipo y cinco paratipos, procedentes de "Massif du Day, Parc National, 1400 m", de la República de Djibuti, al norte de Somalia (Bologna, 1990: 391).

El estudio taxonómico de los integrantes del subgénero Eurymeloe resulta especialmente complicado por la existencia de una gran variabilidad intraespecífica. Esta variabilidad resulta muy difícil de interpretar como consecuencia de la escasez de ejemplares en las colecciones científicas y su dificultad de localización en el campo (Bologna, 1988, 1990, 1991; Ruiz \& García-París, 2009). El caso del complejo de $M$. saharensis es aún más extremo y hasta el momento las escasas interpretaciones taxonómicas se limitan a la sugerencia de una posible sinonimia entre $M$. saharensis y $M$. marianii (Bologna, 1990: 393; Bologna, 2008b: 48; Bologna et al., 2008).

En este trabajo, a partir del estudio de los tipos de $M$. saharensis y M. otini y de material adicional adscribible al complejo de $M$. saharensis, revisamos el estatus taxonómico de las especies del grupo y establecemos nuevas sinonimias. El estudio del material disponible permite considerar los diferentes rasgos diagnósticos señalados para $M$. saharensis, M. otini y $M$. marianii dentro de la plasticidad fenotípica de una única especie, $M$. saharensis, que a diferencia de lo considerado previamente, presenta una amplia distribución geográfica (Fig. 1). 


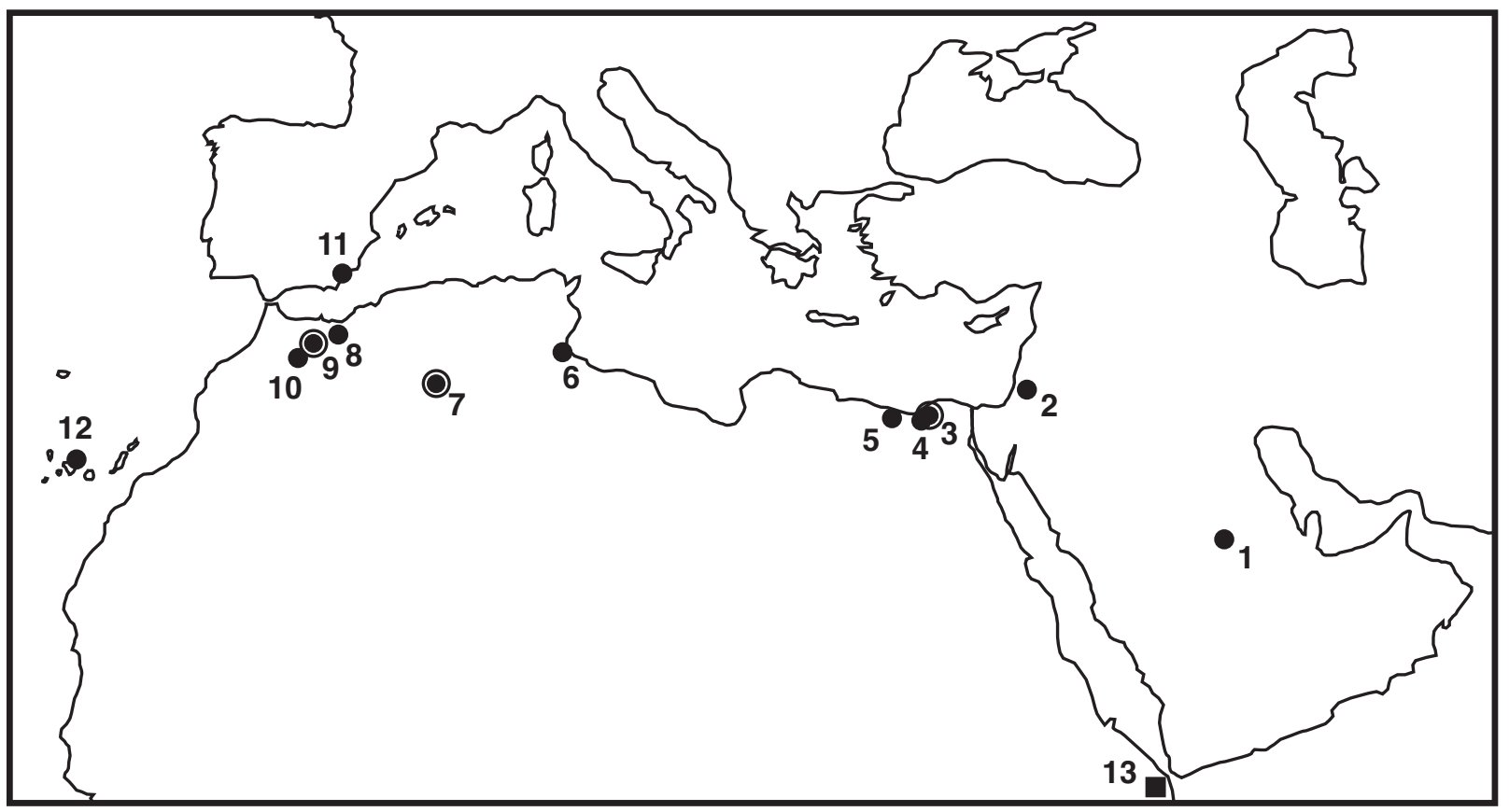

Fig. 1.- Mapa esquemático con las localidades conocidas de Meloe (Eurymeloe) saharensis (1-12) y M. (E.) vignai (13). 1.Khashm Khafs, Arabia Saudí; 2.- Qumran (=Kumran), Israel; 3.- Alejandría, Egipto (localidad tipo de M. marianii); 4.- Ikingi Mariout, Egipto; 5.- Marsa Matrouth (= Marsa Matruht), Egipto; 6.- Matmata, Túnez; 7.- Ghardaia, Argelia (localidad tipo de M. saharensis); 8.- Oujda, Marruecos; 9.- Guercif, Marruecos (localidad tipo de M. otini); 10.- Outat-el-Hajj, Marruecos; 11.- Águilas, España; 12.- Monte Aguirre, Tenerife, España; 13.- Massif du Day, Djibuti (localidad tipo de M. vignai).

Fig. 1.- Map including all known localities where Meloe (Eurymeloe) saharensis (1-12) or M. (E.) vignai (13) have been recorded: 1.- Khashm Khafs, Saudi Arabia; 2.- Qumran (=Kumran), Israel; 3.- Alexandria, Egypt (type locality for M. marianii); 4.- Ikingi Mariout, Egypt; 5.- Marsa Matrouth (= Marsa Matruht), Egypt; 6.- Matmata, Tunisia; 7.- Ghardaia, Algeria (type locality for M. saharensis); 8.- Oujda, Morocco; 9.- Guercif, Morocco (type locality for M. otini); 10.- Outat-el-Hajj, Morocco; 11.- Águilas, Spain; 12.- Monte Aguirre, Tenerife, Spain; 13.- Massif du Day, Djibuti (type locality for M. vignai).

\section{Material estudiado}

El estudio taxonómico de las especies del complejo de $M$. saharensis se basa en el examen de la morfología externa de nueve ejemplares, que incluyen el material tipo de $M$. saharensis, $M$. otini y M. vignai (Fig. 1) y en el estudio de la genitalia de ejemplares representativos. Las colecciones e instituciones en las que se encuentra depositado este material son: Magyar Természettudományi Múzeum (HMNH, Budapest); Muséum National d'Histoire Naturelle (MNHN, París); Museo Civico di Storia Naturale (MSNM, Milán); Museu de Zoologia (MZB, Barcelona); y la colección de Marco A. Bologna (CB, Dipartimento di Biologia Ambientale, Università Roma Tre, Roma). El material estudia- do del complejo taxonómico de $M$. saharensis es el que se relaciona a continuación:

a) ARGELIA: Holotipo de Meloe saharensis: 1 웅 etiquetada: "Ghardaia (Mzab), Sud Algerien" (etiqueta blanca, impresa), "Meloe saharensis Chob., Type" (etiqueta blanca, manuscrita), "Type" (etiqueta impresa, roja) (MNHN) (Fig. 1: 7).

b) TÚNEZ: 19 : “Tunisia, Matmata, 15.II.1997, Pierutti leg." (CB) (Fig. 1: 6).

c) ESPAÑA: 10": "Aguilas, (Murcia). G. Schramm” / "Baudueri Gren., Dr. Z. Kaszab det." (HMNH) (Fig. 1: 11).

d) EGIPTO: 10": "Marsa Matrouth, 17.3.933, Egitto, C. Koch" / "Meloe (Eurymeloe) marianii Kaszab, M. Bologna, det. 1986" (CB) (Fig. 1: 5).

e) EGIPTO: 1 우 : "Ikingi Mariout, 17.3.935, W. Wittmer leg." (MSNM) (Fig. 1: 4).

f) ISRAEL: $10^{7}$ : "Qumran, 18.2.1996, V. Chikutanov leg." (CB) (Fig. 1: 2). 
g) MARRUECOS: Holotipo de Meloe otini (por designación original): $10^{\pi}$, etiquetado: "Plaine de Guercif, 22.1.39, Otin" (etiqueta blanca, impresa) "Meloe otini Peyerimhoff, Type" (etiqueta blanca, manuscrita), "Type" (etiqueta impresa, roja) (MNHN) (Fig. 1: 9).

h) ESPAÑA (Islas Canarias): 10": "Monte Aguirre, Tenerife" (MZB) (Fig. 1: 12).

i) REPÚBLICA DE DJIBUTI: 1 Paratipo de Meloe vignai: 1 O , etiquetado: "Terr. Franç. des Afars et des Issas, Mission Balachowsky-Manier 1972"; "Massif du Day, Parc National, 1400 m, 24.XI.1972" (etiquetas blancas, impresas); "Meloe (Eurymeloe) n. sp. ?, Pardo Alcaide det. 1977" (etiqueta blanca, manuscrita e impresa); "Paratypus, Meloe (Eurymeloe) vignai n. sp., M. Bologna det. 1990" (etiqueta roja, manuscrita e impresa) (CB) (Fig. 1: 13).

\section{Resultados y discusión}

CARACTERIZACIÓN MORFOLÓGICA Y VARIABILIDAD

Las descripciones originales de M. saharensis, M. otini y $M$. marianii presentan un conjunto de caracteres que permiten atribuir cada una de ellas al subgénero Eurymeloe y a su vez separarlas de las especies mejor caracterizadas de los diferentes grupos dentro del subgénero, pero en ningún caso permiten diferenciarlas entre sí.

La descripción más detallada de una de estas especies es la efectuada por Bologna (1988) referida a $M$. marianii. A esta descripción, se pueden añadir los siguientes caracteres obtenidos a partir del material estudiado: placa mesosternal con numerosas arruguitas transversas y borde del margen posterior netamente engrosado y levantado, prolongada hacia atrás a modo de apófisis subtriangular situada entre la base de las mesocoxas; metasternon escotado en forma de $U$ en su margen posterior, entre las metacoxas; artejos del tarso con una agrupación de pelos negros o castaño oscuros cortos, robustos y semierectos en el extremo anterior de su cara inferior, donde ocupan entre $1 / 3$ y $1 / 5$ de la superficie ventral, que pueden reducirse a pelos alineados, sin formar agrupaciones conspicuas, en el primer y último artejos así como en la mayor parte de éstos en los ejemplares de menor tamaño; valvífera de la hembra relativamente ancha, un poco estrechada en su región proximal, el estilo corto y grueso, con escasos pelos rojizos (Fig. 4).

Utilizando esta descripción extendida como referencia y comparándola con cada uno de los ejemplares estudiados, o en su defecto con las descripciones originales de cada una de las especies mencionadas, no hemos identificado ningún carác- ter constante que separe estos taxones, ya que las variaciones observadas se encuadrarían dentro de los límites de variabilidad individual o interpoblacional de las especies del subgénero Eurymeloe. Sin embargo, algunos de los caracteres estudiados muestran un importante grado de plasticidad que merece ser discutido, ya que por ejemplo en el caso de $M$. otini, el caracter indicado en la descripción original como: escutelo prominente, elevado, hinchado y brillante (Peyerimhoff, 1949), fue sin duda determinante a la hora de establecer dicha especie. Los diferentes caracteres se discuten a continuación:

a) Surco longitudinal de la cabeza y pronoto. El holotipo de M. saharensis, la hembra de Matmata y el macho de Tenerife, presentan un surco longitudinal profundo en la frente y la depresión media del pronoto también asurcada. En otros especímenes esta depresión se marca de forma mucho más débil; asimismo, se observa cierta variabilidad en el surco medio frontal de la cabeza, que puede ser más o menos impreso e incluso estar casi borrado. En otras especies de Eurymeloe del grupo de M. rugosus, como M. ganglbaueri Apfelbeck, 1905, M. murinus Brandt \& Erichson, 1832 y M. mediterraneus G. Müller, 1925 se observa una elevada variabilidad en los mencionados caracteres (Bologna, 1988, 1991; obs. pers.).

b) Estructura del metanoto. Tanto el holotipo de $M$. otini como el ejemplar de Tenerife presentan lo que a primera vista parece un escudete prominente y abultado (Fig. 2), tal como señaló Peyerimhoff (1949). Este autor refiere este carácter como diagnóstico de la especie y por ello la compara con M. scutellatus Reitter, 1895, descrita de Transcaucasia (Azerbaiyán), de la que se separa por presentar ésta una coloración distinta, de tono negro plomizo poco brillante, con escutelo bien visible amarillo-rojizo y región distal de los fémures con un anillo amarillo-rojizo -negro brillante con apéndices tornándose castaño rojizo en $M$. saharensis-; pilosidad constituida por pelos largos y muy finos - cortos y castaño rojizos en $M$. saharensis-; cabeza subcuadrada -redondeada en M. saharensis- y pronoto netamente más transverso en la primera, arriñonado y con los lados hacia delante algo ensanchados (Reitter, 1895; Escherich, 1896). 

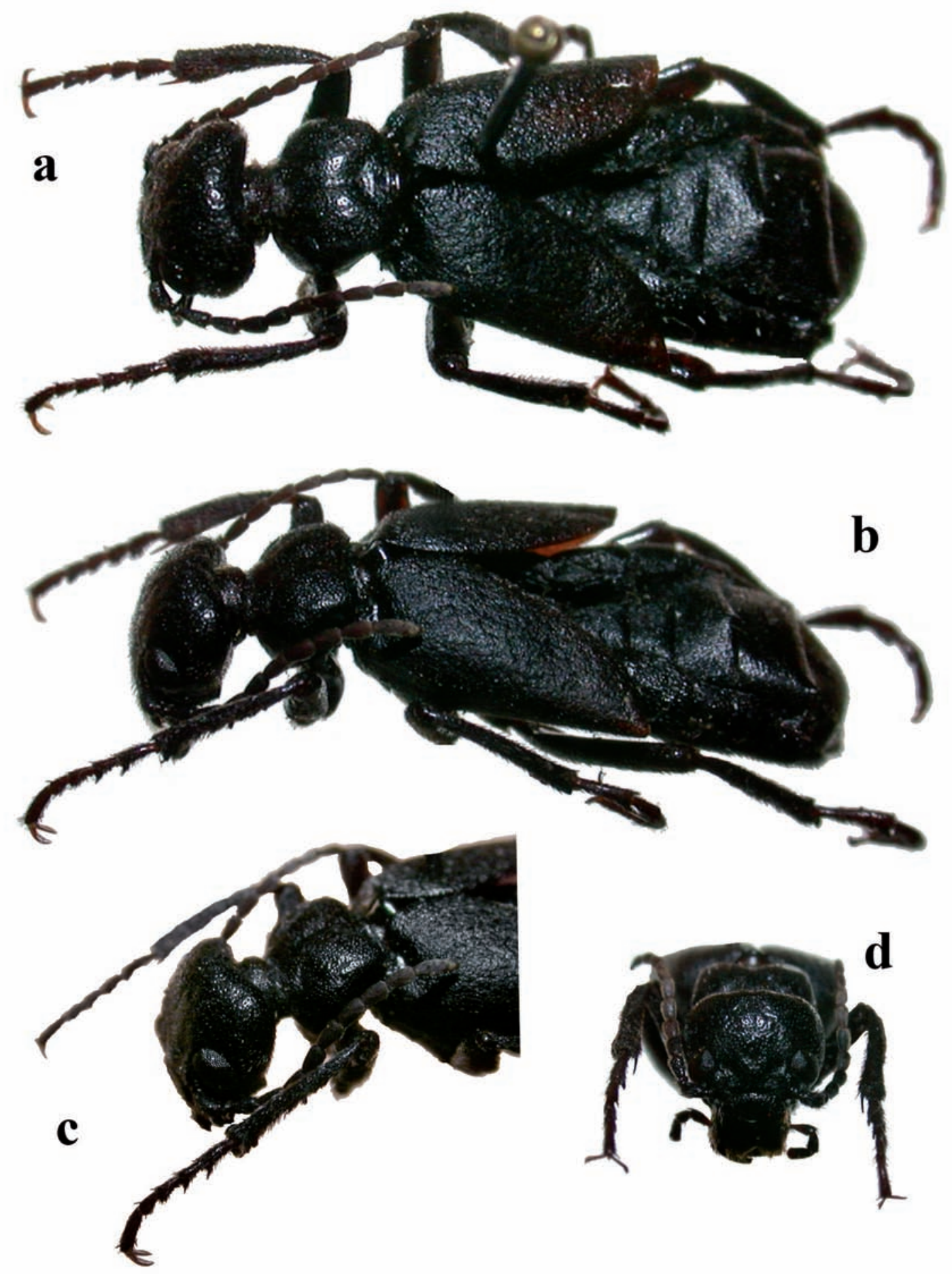

Fig. 2.- Hábitus de Meloe (Eurymeloe) saharensis (Monte Aguirre, Tenerife, España): a) vista dorsal; b) lateral; c) cabeza y torax en visión dorsolateral; d) cabeza frontal.

Fig. 2.- Habitus of Meloe (Eurymeloe) saharensis (Monte Aguirre, Tenerife, Spain): a) dorsal view; b) lateral view; c) head and thorax in dorsolateral view; d) head in frontal view. 

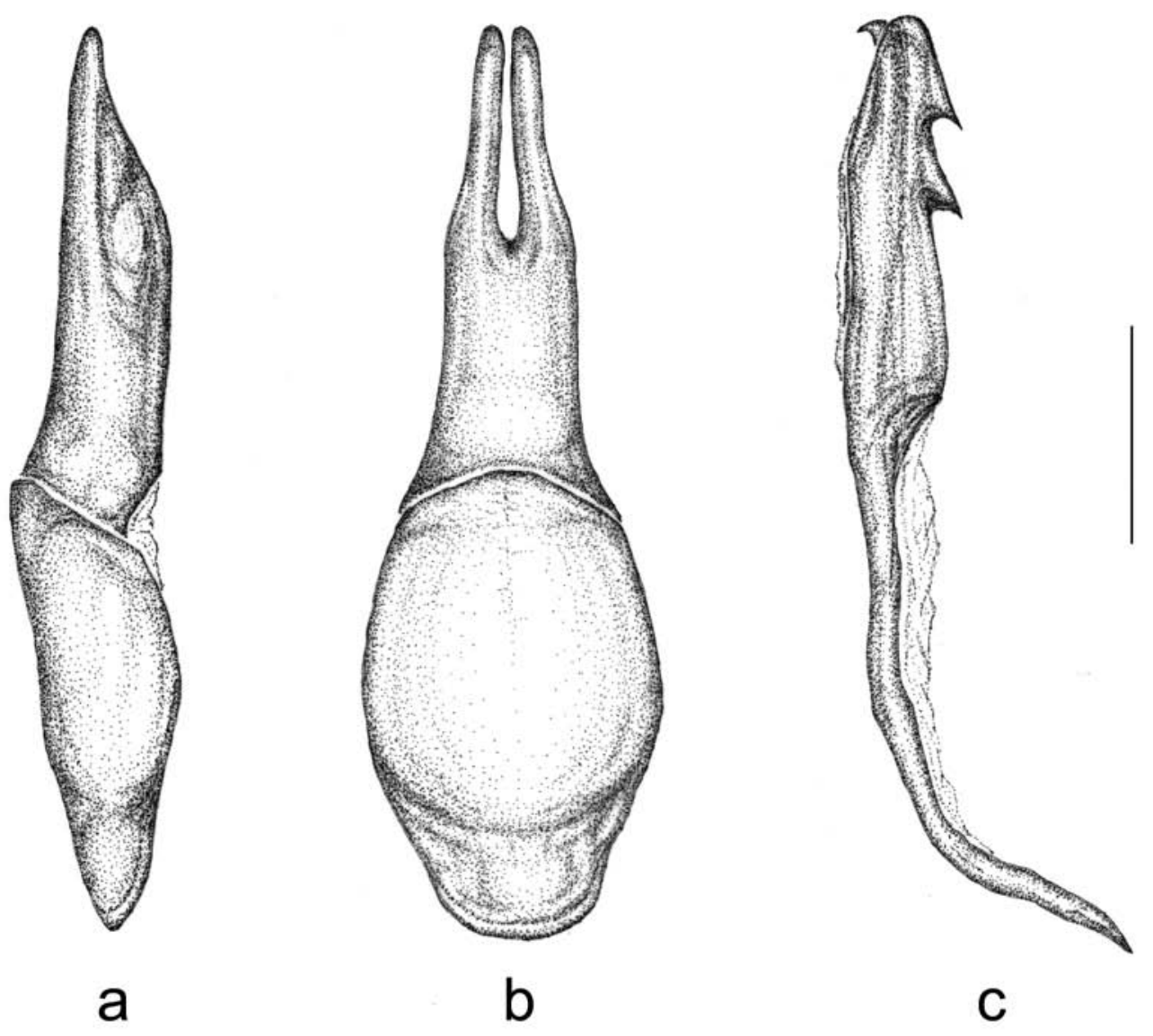

Fig. 3.- Genitalia masculina de Meloe (Eurymeloe) saharensis (Águilas, España): a) tegmen, en visión lateral; b) tegmen, en visión dorsal; c) lóbulo medio, en visión lateral. Escala: 0.5 mm. (J.L. Ruiz del.).

Fig. 3.- Male genitalia of Meloe (Eurymeloe) saharensis (Águilas, Spain): a) tegmen, lateral view; b) tegmen, dorsal view; c) median lobe, lateral view. Scale bar: $0.5 \mathrm{~mm}$. (J.L. Ruiz del.).

Este "escudete" particular no es una expansión posterior del mesonoto sino que corresponde al metanoto, de contorno hemielíptico y dirigido hacia arriba posteriormente, asomando en su totalidad entre la base de los élitros. Los élitros de estos ejemplares se encuentran muy separados y sin imbricar en la base, por lo que no llegan a cubrir el metanoto, que en las especies de Meloe se encuentra muy reducido en correspondencia con su apterismo. Beauregard (1890: 20-21) denomina a esta pieza "arceau tergal" del metatórax. Por tanto, esta prominencia no corresponde a un verdadero escudete o escutelo [expansión media posterior del mesonoto, generalmente subtriangular o hemielipsoi- dal; véase Alonso Zarazaga \& Mansilla Castrillo (1988)] como refiere Peyerimhoff (1949), pues el borde posterior del mesonoto es recto y no prolongado hacia atrás en los ejemplares estudiados, tal como indican Pinto \& Selander (1970: 107) entre los caracteres que definen al subgénero Eurymeloe.

En otros dos ejemplares (Águilas; Marsa Matrouth) también se observa, en el espacio existente entre los márgenes internos de los élitros a nivel basal, un metanoto prominente y brillante, aunque en estos ejemplares el metanoto se encuentra cubierto parcialmente (en su porción posterior) por la base elitral, tratándose de un estado intermedio entre fenotipos con el 


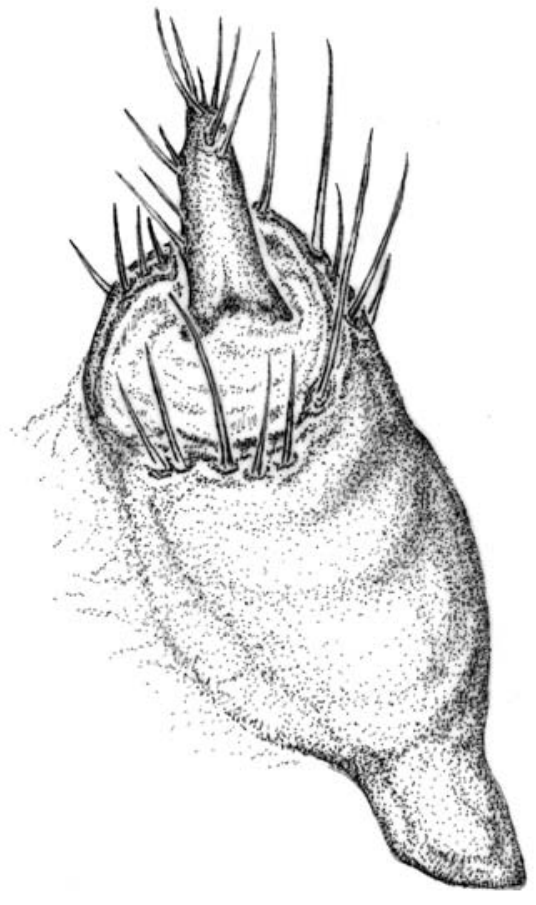

Fig. 4.- Valvífera y estilo de la hembra de Meloe (Eurymeloe) saharensis (Matmata, Túnez). Escala: 0.3 mm (J.L. Ruiz del.).

Fig. 4.- Female valvifer and stylus of Meloe (Eurymeloe) saharensis (Matmata, Tunisia). Scale bar: 0. $3 \mathrm{~mm}$ (J.L. Ruiz del.).

metanoto libre y totalmente visible (holotipo de M. otini y ejemplar de Tenerife) y aquellos que lo muestran casi totalmente cubierto y apenas perceptible (resto de ejemplares examinados). Aunque el tamaño de muestra es muy pequeño, la prominencia del metanoto parece mucho más visible en los ejemplares de mayor tamaño corporal. La existencia de variabilidad en la exposición del metanoto, con estados intermedios entre los extremos, y la ausencia de un patrón geográfico claro en la expresión de dicha variabilidad, sugieren que el grado de exposición de la prominencia metatorácica no tiene relevancia taxonómica dentro del grupo de M. saharensis.

c) Talla. La longitud total del conjunto de ejemplares estudiados está comprendida entre 11,2-16,3 $\mathrm{mm}$ (media $=13,4 \mathrm{~mm} ; \mathrm{n}=8$; el tamaño de los holotipos de $M$. saharensis, $13 \mathrm{~mm}$, y de $M$. otini, $15 \mathrm{~mm}$, se encuadra en el rango de variabilidad detectado; el ejemplar de mayor tamaño estudiado es el de Tenerife: 16,3 mm) (Fig. 2). La relación "anchura máxima/longitud" del pronoto varía entre 1,28 y 1,36 (media $=1,32 ; n=6)$. Las antenas son un poco más largas en los machos, siendo a su vez de aspecto más robusto en los especímenes de mayor tamaño.

d) Otros caracteres. La coloración de las antenas, tarsos y extremo de las tibias está más o menos oscurecida, pero siempre es de un tono castañorojizo. La cabeza y pronoto parecen presentar el punteado más denso e impreso en los ejemplares de mayor tamaño (especialmente en el ejemplar de Tenerife). Los terguitos abdominales presentan punteado más denso en los ejemplares de mayor tamaño, en los que además el tegumento entre los puntos es subrugoso. Además, existen ligeras variaciones en la amplitud y profundidad de las depresiones anterolaterales del pronoto; en la escotadura basal del pronoto, que puede estar más o menos marcada; en la longitud de los lóbulos paramerales de la genitalia masculina; en la anchura de la falobase; y en el tamaño y separación de los dientes ventrales del lóbulo medio, así como en su alejamiento del ápice (en la Fig. 3 se ilustra la genitalia del ejemplar ibérico estudiado).

Meloe vignai se separa de $M$. saharensis, $M$. otini y $M$. marianii por presentar la primera una talla media mayor (rango comprendido entre 9.6-17 $\mathrm{mm}$ ), el tegumento corporal más brillante, la cabeza y el pronoto con punteado más grueso y marcado y tegumento con micropuntos difusos, el surco medio longitudinal de cabeza y la depresión frontal más profundos, los ojos más convexos, los artejos de las antenas netamente más robustos, los lados del pronoto subparalelos, la depresión discal del pronoto distinta y la rugosidad elitral más fuerte (Bologna, 1990). La genitalia masculina es similar a la de $M$. saharensis, de la que se distingue sólo por leves diferencias: lóbulos paramerales más cortos en visión lateral, vaina parameral más ancha en la base en visión dorsal y lóbulo medio un poco más ancho en su mitad distal en visión lateral (véase Bologna, 1990: 393, figs. 31c, d, e). Bologna et al. (2008) sugieren que la escasa diferenciación morfológica entre $M$. saharensis y $M$. vignai sería concordante con una separación reciente entre ambos taxones por un evento de dispersión pleistocénico desde la Península Arábiga. 
POSICIÓN TAXONÓMICA

Teniendo en cuenta lo expuesto en el epígrafe anterior, consideramos que $M$. saharensis, M. otini y M. marianii son formas de un único taxón específico, por lo que establecemos formalmente la sinonimia, ya apuntada parcialmente por Bologna (1990, 1991, 2008a), correspondiendo la prioridad nomenclatural a la primera de ellas, de conformidad con el artículo 23 del Código Internacional de Nomenclatura Zoológica (CINZ, 2000). Por lo tanto, la composición del complejo de Meloe saharensis quedaría limitada a dos especies. El listado sinonímico quedaría como sigue:

Meloe (Eurymeloe) saharensis Chobaut, 1898

Meloe saharensis Chobaut, 1898: 86

Meloe otini Peyerimhoff, 1949: 275 syn. nov.

Meloe (Eurymeloe) marianii Kaszab, 1983: 184 syn. nov.

Meloe (Eurymeloe) vignai Bologna, 1990

Meloe (Eurymeloe) vignai Bologna, 1990: 391

Bologna (1990) adscribe el complejo de especies de M. saharensis al grupo de M. (Eurymeloe) rugosus y, dentro de éste, provisionalmente al subgrupo B o subgrupo de Meloe (Eurymeloe) murinus Brandt \& Erichson, 1832 (sensu Bologna, 1988). Las especies del subgrupo de M. murinus se caracterizan por presentar la pilosidad corporal amarillenta o blanquecina, el tegumento corporal negro o negro-grisáceo opaco, el punteado de la cabeza fino y muy denso y la rugosidad elitral reducida (Bologna, 1988, 1991; Ruiz \& García-París, 2009). Sin embargo, la conjunción de los caracteres propios de este complejo, tales como su aspecto general estilizado, con patas muy largas (especialmente los tarsos) de tonos castaño rojizos, el tegumento corporal negro sedoso casi brillante, la pilosidad corporal corta y en su casi totalidad castaño rojiza, la forma redondeada de la cabeza, el pronoto muy poco transverso, el punteado de la cabeza y pronoto más bien grueso y poco denso, y los élitros con rugosidad elitral muy débil, lo independizan tanto del subgrupo de $M$. murinus como del subgrupo de M. rugosus (sensu Bologna, 1988). Por todo ello consideramos que Meloe saharensis y M. vignai deberían incluirse en un subgrupo independiente dentro del grupo de especies de M. rugosus: el subgrupo de $M$. saharensis.

El ejemplar ibérico (Águilas, provincia de Murcia), conservado en el $\mathrm{HMNH}$, fue determinado como Meloe (Eurymeloe) baudueri Grenier, 1863 por Zoltán Kaszab, autor que, precisamente, describió años más tarde M. marianii. Meloe baudueri, integrada en el subgrupo de $M$. murinus (sensu Bologna, 1988) y distribuida por el suroeste de Francia, España, Marruecos y Argelia (Bologna, 2008a), se separa fácilmente de M. saharensis, entre otros caracteres, por presentar la primera la pilosidad corporal amarillo-blanquecina, el tegumento corporal negro-grisáceo opaco, el pronoto netamente más ancho y con macroescultura distinta, y el punteado de cabeza y pronoto netamente más denso y fino (Bologna, 1988, 1991; Ruiz \& García-París, 2009).

Por otra parte, la localidad que reza en la etiqueta del ejemplar de Tenerife, "Monte Aguirre", es coincidente con la localidad-tipo de Meloe (Eurymeloe) fernandezi Pardo Alcaide, 1951. Meloe fernandezi se separa fácilmente de M. saharensis por presentar la primera un tamaño mayor (13-24 mm); la coloración del cuerpo, apéndices y de toda la pilosidad es negra; el pronoto netamente menos transverso, con la escultura del tegumento particular, presentando estrías y arrugas lineales paralelas entre sí, onduladas, formando algunos remolinos, y élitros con rugosidad fina pero fuertemente marcada, con las rugosidades, brillantes y en zig-zag longitudinal (Pardo Alcaide, 1951; García et al., 1993). Esta especie es un endemismo macaronésico conocido hasta el momento de las islas de La Palma y Tenerife (Pardo Alcaide, 1951; Bologna, 1988, 1994; Bologna \& Marangoni, 1990; García et al., 1993; Machado \& Oromí, 2000; García, 2008).

\section{DisTRIBUCIÓN Y NOTAS AUTOECOLÓGICAS}

Meloe saharensis muestra una amplia distribución, desde Israel y Arabia Saudí hasta las Islas Canarias (Tenerife), con presencia en el sureste de la Península Ibérica y en la mayor parte de los países norteafricanos (Egipto, Túnez, Argelia y Marruecos) (Fig. 1) (Tabla 1). Su extensa área de distribución contrasta con la escasez de registros para la especie, un total de 12 en su mayor parte muy distanciados entre sí (Tabla 1). Los registros de Águilas (Murcia, España) y Tenerife (España, Islas Canarias) constituyen los primeros para el continente europeo y la región macaronésica respectivamente, el de Matmata, en Túnez centrooriental, es igualmente el primero para este país y el de la depresión del Mar Muerto (Qumran, desierto de Judea) constituye el primero concreto para Israel. 
Tabla 1.- Localidades conocidas de Meloe (Eurymeloe) saharensis. Precipitación media anual obtenida de: (1) Ghazanfar \& Fisher (1998); (2) Goldreich (2003); (3) Le Houerou (1989); (4) Rivas-Martínez (1987); (5) Del Arco et al. (2006).

Tabla 1.- Known records for Meloe (Eurymeloe) saharensis. Average annual rainfall sources: (1) Ghazanfar \& Fisher (1998); (2) Goldreich (2003); (3) Le Houerou (1989); (4) Rivas-Martínez (1987); (5) Del Arco et al. (2006).

\begin{tabular}{|c|c|c|c|c|c|}
\hline Localidad & País & $\begin{array}{l}\text { Provincia/Región } \\
\text { administrativa }\end{array}$ & $\begin{array}{l}\text { Coordenadas } \\
\text { geográficas }\end{array}$ & Altitud & $\begin{array}{l}\text { Precipitación media } \\
\text { anual (fuente) }\end{array}$ \\
\hline $\begin{array}{l}\text { Khashm Khafs } \\
\text { (Kaszab, 1983) }\end{array}$ & Arabia Saudí & Mintaqad ar Ryad & $\begin{array}{l}25.516^{\circ} \mathrm{N} \\
46.45^{\circ} \mathrm{E}\end{array}$ & $730 \mathrm{~m}$ & $50-100 \mathrm{~mm}(1)$ \\
\hline Qumran (= Kumran) & Israel & Cisjordania & $\begin{array}{l}31.743351^{\circ} \mathrm{N} \\
35.459747^{\circ} \mathrm{E}\end{array}$ & $-350 m$ & $100 \mathrm{~mm}(2)$ \\
\hline $\begin{array}{l}\text { Alejandria } \\
\text { (Kaszab, 1983) }\end{array}$ & Egipto & Muhafazat al Iskandariyan & $\begin{array}{l}31.198^{\circ} \mathrm{N} \\
29.919^{\circ} \mathrm{E}\end{array}$ & $5 \mathrm{~m}$ & 191 mm (3) \\
\hline $\begin{array}{l}\text { Ikingi Mariout } \\
\text { (Bologna, 1988) }\end{array}$ & Egipto & Muhafazat al Iskandariyan & $\begin{array}{l}30.992^{\circ} \mathrm{N} \\
29.766^{\circ} \mathrm{E}\end{array}$ & $23 \mathrm{~m}$ & 190 mm (3) \\
\hline $\begin{array}{l}\text { Marsa Matrouth } \\
\text { (= Marsa Matruht) } \\
\text { (Bologna, 1988) }\end{array}$ & Egipto & Muhafazat Matruht & $\begin{array}{l}31.35^{\circ} \mathrm{N} \\
27.233^{\circ} \mathrm{E}\end{array}$ & $40 \mathrm{~m}$ & 123 mm (3) \\
\hline Matmata & Túnez & Gouvernorat de Gabes & $\begin{array}{l}33.55^{\circ} \mathrm{N} \\
9.966^{\circ} \mathrm{E}\end{array}$ & $402 \mathrm{~m}$ & 239 mm (3) \\
\hline $\begin{array}{l}\text { Ghardaia } \\
\text { (Chobaut, 1898) }\end{array}$ & Argelia & Wilaya de Relizane & $\begin{array}{l}32.483^{\circ} \mathrm{N} \\
3.666^{\circ} \mathrm{E}\end{array}$ & $572 \mathrm{~m}$ & 68 mm (3) \\
\hline $\begin{array}{l}\text { Oujda } \\
\text { (Kocher, 1954, 1956) }\end{array}$ & Marruecos & Provincia de Oujda & $\begin{array}{l}34.68^{\circ} \mathrm{N} \\
1.91^{\circ} \mathrm{O}\end{array}$ & $568 \mathrm{~m}$ & 335 mm (3) \\
\hline $\begin{array}{l}\text { Guercif } \\
\text { (Peyerimhoff, 1949) }\end{array}$ & Marruecos & Provincia de Taza & $\begin{array}{l}34.23^{\circ} \mathrm{N} \\
3.36^{\circ} \mathrm{O}\end{array}$ & 378 m & 199 mm (3) \\
\hline $\begin{array}{l}\text { Outat-el-Hajj } \\
\text { (Kocher, 1954, 1956) }\end{array}$ & Marruecos & Provincia de Boulemane & $\begin{array}{l}33.34^{\circ} \mathrm{N} \\
3.69^{\circ} \mathrm{O}\end{array}$ & $785 \mathrm{~m}$ & 157 mm (3) \\
\hline Águilas & $\begin{array}{l}\text { España } \\
\text { (Península lbérica) }\end{array}$ & Provincia de Murcia & $\begin{array}{l}37.400^{\circ} \mathrm{N} \\
1.583^{\circ} \mathrm{O}\end{array}$ & $10 \mathrm{~m}$ & 177 mm (4) \\
\hline Monte Aguirre & $\begin{array}{l}\text { España } \\
\text { (Islas Canarias) }\end{array}$ & Provincia de Tenerife & $\begin{array}{l}28.522^{\circ} \mathrm{N} \\
16.269^{\circ} \mathrm{O}\end{array}$ & $551 \mathrm{~m}$ & $300-400 \mathrm{~mm}(4,5)$ \\
\hline
\end{tabular}

Como ya apuntó Bologna (1988, para M. marianii) y se puede inferir de la práctica totalidad de localidades conocidas (véase Tabla 1), M. saharensis se comporta como una especie erémica propia de regiones desérticas, semidesérticas y semiáridas, tanto en áreas costeras o subcosteras como interiores, con un rango altitudinal extendido desde el nivel del mar (Águilas, Alejandría y otras localidades egipcias) hasta $785 \mathrm{~m}$ de altitud (Outat-el-Hajj), con excepción de la estación de Qumran, situada a unos $350 \mathrm{~m}$ bajo el nivel del mar (margen noroccidental de la depresión del Mar Muerto). La precipitación media anual de las estaciones que integran su área de ocupación (sensu UICN, 2001) se indican en la Tabla 1, encuadrándose en ombrotipos hiperárido superior, árido (superior e inferior) y semiárido (superior e inferior) (véase Rivas Martínez, 1987; Le Houerou, 1989; Ghazanfar \& Fisher, 1998; Goldreich, 2003).

En cuanto a la localidad canaria, en la etiqueta (manuscrita) del ejemplar examinado sólo reza "Monte Aguirre, Tenerife", sin precisión del lugar concreto de captura, fecha, ni del colector. Este topónimo se refiere al monte de utilidad pública $\mathrm{n}^{\circ}$ 44 con idéntica denominación ("Monte de Aguirre"), situado unos $6,5 \mathrm{~km}$ en línea recta al norte de Sta. Cruz de Tenerife, perteneciente a su término municipal, y se ubica en el ámbito del 
espacio natural protegido "Parque Rural de Anaga" (Macizo de Anaga), en el extremo noreste de la isla. El territorio que constituye el "Monte de Aguirre" (superficie aproximada: $2 \mathrm{~km}^{2}$ ) está ocupado en su mayor parte por vegetación de tipo laurisilva o monteverde, tanto de tipo seco (fayal-brezal, serie de vegetación potencial: Visneo mocanerae-Arbuto canariensis S.) como húmedo (Lauro novacanariense-Perseo indicae $S$.), que viene determinada por una pluviosidad y precipitación horizontal (nieblas) elevadas, por lo que aparentemente, no constituirían hábitats propicios para la especie; esta tipología de hábitat sería la propia de M. fernandezi (García et al., 1993; García, 2008). Sin embargo, en cotas más bajas de la porción del macizo de Anaga, en laderas y barrancos de orientación meridional (Barranco de Tahodio) y a menos de $1 \mathrm{~km}$ hacia el sur (en medición digital), aparecen extensas formaciones de tabaibal-cardonal y de sabinalacebuchal-almacigal, pues en un espacio muy corto disminuyen notablemente la pluviosidad y las criptoprecipitaciones (entre 300-400 $\mathrm{mm}$ de precipitación media anual), como es habitual en esta isla. Estas últimas zonas presentan características ambientales y hábitats tipológicamente semejantes, en términos generales, a los del resto del área de ocupación de la especie.

Dada la presencia de $M$. saharensis en la isla de Tenerife, es posible la existencia de núcleos poblacionales de la especie en las islas orientales del archipiélago canario (Lanzarote, Fuerteventura, e incluso en Gran Canaria), que albergan hábitats áridos y semiáridos propicios para la misma (RivasMartínez, 1987; Del Arco et al., 2006) y en las que habitan otros Meloidae propios de ambientes erémicos, como Meloe (Mesomeloe) coelatus Reiche \& Saulcy, 1857 y Meloe (Meloe) aegyptius subcyaneus Wollaston, 1864 (Bologna, 1994; Machado \& Oromí, 2000).

El ejemplar de Águilas fue capturado por George Schramm (1870-1956), entomólogo que colectó intensamente en España en las dos primeras décadas del siglo XX (especialmente en la provincia de Murcia, al residir unos años en Cartagena). Aunque en la etiqueta de localidad no se consigna la fecha de captura del espécimen, éste hubo de colectarse en el mencionado periodo, por lo que el único registro ibérico de la especie dataría de hace más de 80 años, sin que se disponga de datos más recientes. Desde entonces la especie no ha vuelto a ser localizada en la región. La localidad de Águilas se sitúa desde el punto de vista fitocorológico en la provincia murciano-almeriense, sector almeriense, en el horizonte bioclimático termomediterráneo inferior (Rivas Martínez et al., 2002), con un ombrotipo semiárido inferior y vegetación xérica adscribible mayoritariamente a las series termomediterránea almeriense semiárida-árida del azufaifo (Ziziphus lotus), Zizipheto loti S., y termomediterránea inferior almeriense semiárido-árida del cornical (Periploca laevigata), Mayteno europaei-Periploceto angustifoliae S. (Alcaraz \& Peinado, 1987; Valle, 2003). Se trata de una de las localidades que registra menor precipitación media anual $(177 \mathrm{~mm})$ en la Península Ibérica (Rivas-Martínez, 1987; Alcaraz \& Peinado, 1987). Estas características bioclimáticas se extienden a otras regiones del sureste ibérico semiárido, especialmente en la porción oriental de la provincia de Almería (Andalucía).

Creemos que la especie, aparentemente muy rara y localizada en toda su área de distribución, merece la realización de muestreos exhaustivos en las zonas semiáridas del sureste ibérico y de las Islas Canarias con objeto de determinar la continuidad de su existencia.

\section{Agradecimientos}

Nuestro agradecimiento a Glòria Masò (MZB, Barcelona), Otto Merkl (HMNH, Budapest), Judit Vörös (HMNH, Budapest) y Mercedes Paris (MNCN, Madrid) por las facilidades prestadas para el examen de material de las colecciones del MZB, HMNH y MNCN; a Pepe Fernández (MNCN, Madrid) por su ayuda en la edición del manuscrito; y al Instituto de Estudios Ceutíes, por su continuo apoyo. Agradecemos a un evaluador anónimo y a Antonio Machado la revisión crítica de una versión anterior de este trabajo.

Este trabajo ha contado para su realización con el proyecto CGL2007-64621 del Ministerio de Educación y Ciencia (España), lo que nos ha facilitado el apoyo logístico necesario. La visita de MGP a la colección del Magyar Természettudományi Múzeum (HNHM) en Budapest ha sido financiada por el proyecto Synthesys «European Commission's Research Infrastructure Action».

\section{Referencias}

Alcaraz, F. \& Peinado, M., 1987. España semiárida: Murcia y Almería. In: M. Peinado \& S. Rivas-Martínez (eds.). La vegetación de España. Universidad de Alcalá de Henares, Secretaría General, Servicio de Publicaciones. Alcalá de Henares: 257-281.

Alonso Zarazaga, M. A. \& Mansilla Castrillo, O., 1988. Clave artificial de las familias ibero-baleares y maca- 
ronésicas del Orden Coleoptera L. 1758. Claves para la identificación de la fauna española, 20. Universidad Complutense. Madrid. 67 pp., 12 láms.

Beauregard, H., 1890. Les insectes vésicants. Félix Alcan. París. i-xvi, 1-544, 34 láms.

Bologna, M. A., 1988. Note su Eurymeloe e revisione delle specie euromediterranee del gruppo rugosus (Coleoptera, Meloidae). Fragmenta Entomologica, 20: 233-301.

Bologna, M. A., 1990. Faunistica e zoogeografia dei Meloidae (Coleoptera) della Somalia. Biogeographia, [1988], 14: 293-401.

Bologna, M. A., 1991. Fauna d'Italia. XXVIII. Coleoptera Meloidae. Calderini. Bologna. i-xiv, 541 pp.

Bologna, M. A., 1994. Meloidae from Canary and other macaronesian islands (Coleoptera). Miscel-lània Zoològica, [1992], 16: 73-80.

Bologna, M. A., 2008a. Meloidae. In: I. Löbl \& A. Smetana (eds.). Catalogue of Palaearctic Coleoptera. Vol. 5. Tenebrionoidea. Apollo Books. Stenstrup: 370-412.

Bologna, M. A., 2008b. New Acts and Comments. Meloidae. In: I. Löbl \& A. Smetana (eds.). Catalogue of Palaearctic Coleoptera. Vol. 5. Tenebrionoidea. Apollo Books. Stenstrup: 45-49.

Bologna, M. A., Aloisi, G. \& Marangoni, C., 1989. Nuove osservazioni su Eurymeloe Reitter e descrizioni di larve di I stadio (Coleoptera, Meloidae). Bulletin et Annales de la Société Royale Belge d'Entomologie, 125: 67-75.

Bologna, M. A., Di Giulio, A. \& Pitzalis, M., 2008. Examples of disjunct distributions between Mediterranean and southern or eastern Africa in Meloidae (Coleoptera, Tenebrionoidea). Biogeographia, 28: 8198.

Bologna, M. A. \& Marangoni, C., 1990. Dispersal, dispersion and phoresy in the blister beetle fauna (Coleoptera, Meloidae) of eastern Mediterranean and other islands. Atti dei Convegni "Biogeographical aspects of insularity”, Accademia Nazionale dei Lincei, 85: 345-366.

Chobaut, A., 1898. Description de quelques espèces et variétés nouvelles de coléoptères algériens. Revue d'Entomologie, 17: 74-88.

CINZ (Comisión Internacional de Nomenclatura Zoológica), 2000. Código Internacional de Nomenclatura Zoológica, $4^{a}$ Edición. Museo Nacional de Ciencias Naturales (CSIC) e International Trust for Zoological Nomenclature. Madrid. i-xxix + 156 pp. [Traducción al español de: ICZN, 1999. International Code of Zoological Nomenclature. $4^{\text {th }} \mathrm{ed}$. International Trust for Zoological Nomenclature, c/o The Natural History Museum. London. i-xxix + 306 pp.].

Del Arco, M., Wildpret, W., Pérez de Paz, P. L., Rodríguez, O., Acebes, J. R., García-Gallo, A., Martín,
V. E., Reyes-Betancort, J. A., Salas, M., Bermejo, J. A., González, R., Cabrera, M. V. \& García, S., 2006. Mapa de vegetación de Canarias. GRAFCAN. Santa Cruz de Tenerife. 550 pp, 7 mapas, 1 CD.

Escherich, K., 1896. Meloiden-studien. IV. Theil. Wiener Entomologische Zeitung, 15: 27-30.

García, R., 2008. Distribución de la familia Meloidae (Coleoptera) en la Isla de La Palma. Revista de Estudios Generales de la isla de La Palma, [2007], 3: 363-380.

García, R., Ortega, G. \& Pérez, J. M., 1993. Insectos de Canarias. Excmo. Cabildo Insular de Gran Canaria. Las Palmas de Gran Canaria. [1992]. 418 pp.

Ghazanfar, S. A. \& Fisher, M. (eds.), 1998. Vegetation of the Arabian Peninsula. Series Geobotany, Vol. 25. Kluwer Academic Publishers. Dordrecht. 362 pp.

Goldreich, Y., 2003. The climate of Israel: observation, research and application. Kluwer Academic/Plenum Publishers. New York. i-xxvii, 270 pp.

Kaszab, Z., 1983. Insects of Saudi Arabia. Coleoptera: Fam. Meloidae. A synopsis of the Arabian Meloidae. In: W. Wittmer \& W. Büttiker (eds.). Fauna of Saudi Arabia. Vol. 5. Pro Entomologia c/o Natural History Museum, Basel and Meterorology and Environmental Protection Administration, Jeddah. Basel: 144-204.

Kocher, L., 1954. Prospection entomologique (Coléoptères) de la Moyenne Mouluya. Bulletin de la Société de Sciences naturelles et physiques du Maroc, 34: 263-286.

Kocher, L., 1956. Catalogue commenté des Coléoptères du Maroc. V. Héteromères (Tenebrionides excepts). Travaux de l'Institut Scientifique Chérifien (série Zoologie), 10: 1-107.

Le Houerou, H. N., 1989. Classification écoclimatique des zones arides (s.1.) de l'Afrique du Nord. Ecologia mediterranea, 15(3/4): 95-144, 7 tabs.

Machado, A. \& Oromí, P., 2000. Elenco de los Coleópteros de las Islas Canarias. Instituto de Estudios Canarios, Monografía 70. La Laguna. 306 pp.

Pardo Alcaide, A., 1951. Estudios sobre Meloidae. III. Una nueva especie de Meloe de la isla de Tenerife y comentarios sobre algunos meloideos de la citada isla. Eos, Revista Española de Entomología, 25: 249255.

Peyerimhoff, P. de, 1949. Études et descriptions des coléoptères marocains II. Bulletin de la Société de Sciences naturelles et physiques du Maroc, [194547], 25-27: 248-308.

Pinto, J. D. \& Selander, R. B., 1970. The bionomics of blister beetles of the genus Meloe and a classification of the New World species. Illinois Biological Monographs, 42: 1-222.

Reitter, E., 1895. Bestimmungs-Tabellen der europaïschen Coleopteren Meloidae. I Theil: Meloini. 32. Verlag des Verfassers. Paskau: 1-13. 
Rivas-Martínez, S., 1987. Memoria del mapa de las series de vegetación de España 1:400.000. ICONA. Madrid. 268 pp.

Rivas-Martínez, S., Díaz, T. E., Fernández-González, F., Izco, J., Loidi, J., Lousa, M. \& Penas, A., 2002. Vascular plant communities of Spain and Portugal. Addenda to the syntaxonomical checklist of 2001. Part I. Itinera Geobotanica, 15(1): 5-432.

Ruiz, J. L. \& García-París, M., 2009. Descripción de una especie nueva de Meloe Linnaeus, 1758 del subgénero Eurymeloe Reitter, 1911 (Coleoptera, Meloidae) del norte de Marruecos. Graellsia, 65(2): 91-109.

UICN (Unión Internacional para la Conservación de la Naturaleza), 2001. Categorías y Criterios de las Listas Rojas de la UICN: Versión 3.1. Comisión de Supervivencia de Especies de la UICN. UICN, Gland, Suiza, Cambridge, Reino Unido. ii +33 pp.
Valle, F. (ed.), 2003. Mapa de Series de Vegetación de Andalucía. Rueda S.L. Madrid. 131 pp., 1 mapa escala 1:400.000.

\section{Nota (añadida en pruebas)}

Estando en revisión este trabajo, hemos recibido para estudio (M. Bologna) una serie de seis ejemplares atribuibles a Meloe saharensis procedentes de Italia: isla de Lampedusa: Ponente, 2-I-1985 (M. Pavesi leg.). La isla de Lampedusa se localiza a unos $130 \mathrm{~km}$ de la costa oriental de Túnez, unos $300 \mathrm{~km}$ al noreste de la localidad de Matmata, donde la especie se localizó con anterioridad. Este registro es el primero para Italia y el tercero en territorio de la Unión Europea. 